\title{
APLICACIÓN DE LA TÉCNICA EDUCATIVA APRENDIZAJE BASADO EN PROBLEMAS PARA CAPACITACIÓN A DISTANCIA (E-LEARNING)
}

\author{
(APPLICATION OF THE LEARNING METHOD BASED ON DISTANCE TRAINING PROBLEMS \\ (E-LEARNING))
}

Moisés David Armenta Hernández

Éxito Software (México)

Verónica Salinas Urbina

Fernando Mortera Gutiérrez

Escuela de Graduados en Educación, Tecnológico de Monterrey (México)

\section{RESUMEN}

Este artículo trata sobre el proceso de capacitación en una fábrica de Software para el sector educativo. En un contexto de innovación donde los productos se reinventan constantemente, el reutilizar conocimientos previos y el aprendizaje profundo y autodirigido son fundamentales. Esto orientó la investigación hacia dos técnicas didácticas constructivistas: el aprendizaje colaborativo y el ABP (Aprendizaje Basado en Problemas). Esta investigación de corte cualitativo implementó un estudio de caso para explorar y analizar qué sucede al incorporar en línea a distancia una actividad instruccional basada en la técnica didáctica ABP usando la plataforma educativa "SesWeb". Se recolectaron datos mediante observación virtual, entrevistas cualitativas como herramientas principales, y un cuestionario como herramienta auxiliar. Las evidencias señalan que el modelo ABP 4X4 en modalidad E-learning: a) propicia interacciones para el aprendizaje colaborativo, b) fomenta el aprendizaje profundo y auto dirigido, y c) la investigación individual guía al alumno hacia la resolución del problema.

Palabras clave: e-learning, técnicas didácticas, capacitación corporativa, aprendizaje basado en problemas (ABP), adquisición de conocimiento, enseñanza de tecnología.

\section{ABSTRACT}

This article deals with the training process used in a software company in the education sector. Within the context of innovation, that is, where products are constantly being reinvented, the re-utilization of previous knowledge and deep and self-guided learning are essential. Indeed, this guide is geared towards two constructivist didactic techniques, namely 
collaborative learning and Problem Based Learning (PBL).This qualitative research involved the implementation of a case study that explored and analyzed what happened when we incorporated an instructional activity that is based on the PBL technique using the "SesWeb" educational platform via the online/distance modality. Data was collected via online observations, qualitative interviews (used as primary tools) and a questionnaire (used as an auxiliary tool). The results demonstrate that the 4x4 PBL model in the E-Learning modality: a) facilitates interactions for collaborative learning; b) promotes deep and self-guided learning; and c) that individual research guides students towards problem-solving.

Keywords: e-learning, teaching techniques, corporate training, problem based learning (PBL), knowledge acquisition, educational technology.

La necesidad de llevar la educación a las masas y la carencia de infraestructura para atender las demandas de educación formal y educación continua al ritmo que la sociedad actual demanda han motivado que instituciones educativas y organizaciones corporativas de todo el orbe perciban la educación en línea y a distancia como una opción educativa factible y rentable. Peters (2002, p. 34) menciona: "Nuestro sistema tradicional de educación, con clases y conferencias presenciales, simple y sencillamente no podrá con las tareas que le esperan, el sistema no puede expandirse construyendo más instalaciones físicas, y no puede financiarse”. El análisis costobeneficio realizado por universidades y corporaciones que han incorporado a sus programas educativos la modalidad on-line refleja eficiencia y rentabilidad. Pastore (2002) afirma que el advenimiento y desarrollo del e-learning, en modalidad en línea, refleja la evolución de una sociedad más orientada a la sociedad de conocimiento donde el aprendizaje a distancia se ha convertido en un proceso continuo más que momentáneo. El escenario educativo actual se encuentra inmerso en un proceso de cambio continuo impulsado en gran medida por el surgimiento de una sociedad del conocimiento caracterizada por su aprendizaje constante (Cabero, 2007). Esta sociedad basada en la ciencia y la tecnología precisa de profesionales críticos e innovadores con capacidad de adaptación a situaciones cambiantes y de incertidumbre, y con capacidad para la toma de decisiones y la solución de problemas (Cruz et al., 2012). En este mismo escenario están inmersas instituciones educativas comprometidas a egresar estudiantes críticos, conscientes del valor del aprendizaje autodirigido como factor de éxito en su práctica profesional (GarcíaFamoso, 2005), y organizaciones corporativas que demandan aprender con mayor rapidez para poder sobrevivir en un mundo cada vez más competitivo y complejo (Uden y Beaumont, 2006).

El ritmo frenético con que se reinventan y evolucionan las Tecnologías de la Información y la Comunicación (TIC) convierte al conocimiento de la tecnología 
en un capital intelectual efímero en riesgo constante de volverse obsoleto a corto plazo como consecuencia del surgimiento de tecnologías emergentes, que a su vez requieren de nuevos conocimientos para su implementación y uso. Estos factores convierten la enseñanza de tecnología en un proceso educativo complejo. El tiempo requerido para adquirir conocimientos y aplicarlos en el entorno productivo es una variable crítica en ambientes corporativos. Ehuletche y Le Estefano (2011, pp. 7778) afirman: "[...] los cambios continuos en la tecnología y en la organización del trabajo cuestionan la rigidez de los sistemas de formación dirigidos a ocupaciones específicas". La enseñanza de la tecnología requiere de programas educativos ágiles, que promuevan un aprendizaje constante, crítico y autodirigido. En este contexto, el enfoque de aprendizaje constructivista cobra un carácter estratégico por fomentar el aprovechamiento de los conocimientos previos y el desarrollo de competencias para el aprendizaje activo, necesario para un análisis crítico y reflexivo del conocimiento nuevo, proporcionando elementos para la adopción temprana de tecnologías innovadoras en ambientes corporativos que son materia prima para el desarrollo de productos innovadores que el mercado demanda y que mantienen la continuidad de las empresas. Esta necesidad educativa orientó esta investigación al estudio de técnicas didácticas con enfoque constructivista: el aprendizaje colaborativo y el aprendizaje basado en problemas, aplicados al contexto de capacitación corporativa en una fábrica de Software.

La empresa donde se realiza este estudio de investigación se llama Éxito Software. Fundada en 1988 como un negocio de computación, evoluciona en 1993 a una fábrica de Software especializado en el sector educativo, siendo ServoEscolar (sistema de control escolar y administrativo para escuelas y universidades) y SesWeb (plataforma de servicios académicos y administrativos para instituciones educativas) sus productos más representativos. La misión de Éxito Software es proveer soluciones tecnológicas que potencien los procesos estratégicos, administrativos y operativos en instituciones educativas y en ambientes corporativos. Esta misión expresa un compromiso con el sector educativo, manifestado en la persistencia de la organización por ofrecer sistemas computacionales actualizados, no solo con la funcionalidad que demandan las organizaciones escolares innovadoras, sino también alineados con las novedades tecnológicas emergentes.

\section{PLANTEAMIENTO DEL PROBLEMA}

El proceso de capacitación en el área de producción de software para el sector educativo es una labor permanente que requiere del análisis de las necesidades más puntuales y demandantes del mercado, para que la fuerza de trabajo que labora en 
este tipo de industria esté actualizada y sea competitiva. La necesidad de negocios expresada en la misión de Éxito Software, conduce a una necesidad educativa, ya que mantener actualizados los productos de software para el sector educativo con las tecnologías emergentes requiere de un proceso de aprendizaje constante en el interior de la compañía. Este proceso de capacitación corporativa se realiza en dos sentidos, el primero orientado a los equipos de ingeniería para que adquieran las competencias técnicas necesarias para fabricar software alineado con las tecnologías emergentes y un segundo proceso orientado a informar y capacitar al personal de soporte y distribuidores (empresas que venden, implementan y capacitan los productos desarrollados por la compañía) en las nuevas características y funciones del software liberado al mercado. Para esto, la organización implementó la plataforma Éxito Software University con el propósito de capacitar en línea a distribuidores y personal de soporte técnico.

Un producto de software exitoso demanda no solo competencias técnicas para su fabricación, requiere también entrenar al personal de soporte técnico y distribuidores en la implementación de soluciones y capacitación al usuario final. Esta necesidad educativa ocurre de manera cíclica cada vez que se libera una nueva versión o producto al mercado. Es por eso que Éxito Software necesita encontrar los medios que le permitan disponer de un proceso más ágil y eficiente para la capacitación de su personal de soporte y sus distribuidores, explorando estrategias educativas que acorten la brecha existente entre la adquisición del conocimiento y la aplicación de este nuevo conocimiento en la solución de problemas típicos en la implementación y entrenamiento a usuarios finales. De aquí se desprende la interrogante que condujo este trabajo de investigación:

¿Qué sucede al incorporar la técnica didáctica constructivista Aprendizaje Basado en Problemas (ABP) en un curso de capacitación impartido en línea al personal de atención al cliente (soporte técnico y distribuidores)?

Para resolver esta pregunta de investigación se diseñó una actividad académica donde se aplica la técnica educativa ABP impartida a un grupo en capacitación, explorando los sucesos durante el trabajo académico en línea. El desarrollo de la situación didáctica del ABP explorada también consideró actividades de aprendizaje colaborativo como la discusión en foros y la construcción social-colaborativa de wikis. 


\section{OBJETIVOS DEL ESTUDIO}

El objetivo general de este estudio fue explorar y analizar lo que sucede en un curso de capacitación en plataforma educativa SesWeb cuando se incorpora en línea y a distancia una actividad académica basada en la técnica educativa ABP.

Los objetivos específicos de este estudio fueron los siguientes:

- Explorar qué sucede cuando se aplica en una actividad académica la técnica didáctica ABP al impartir el tema "Módulo de programación de cursos para el aula virtual" en un curso de capacitación en línea usando la plataforma educativa SesWeb.

- Describir el proceso de aprendizaje colaborativo que se lleva a cabo en los equipos de trabajo al aplicar la técnica didáctica ABP en un curso impartido en línea.

- Explorar los beneficios que la técnica educativa ABP aporta para el logro de los objetivos del tema del curso.

- Identificar beneficios adicionales de la técnica didáctica ABP aplicables al contexto corporativo de una fábrica de software.

\section{MARCO TEÓRICO}

Aunque las Tecnologías de la Información y la Comunicación (TIC) raramente son diseñadas con un fin de enseñanza y aprendizaje, estas han detonado una explosión creativa de nuevas maneras de enseñar y aprender (López y Álvarez, 2011). Este hecho transforma a las TIC en un proveedor estratégico del sector educativo, proporciona los medios, herramientas y soluciones que, bien empleados, favorecen la creación de nuevos ambientes de aprendizaje. El surgimiento de la web 2.0 enriqueció los ambientes de aprendizaje en línea al diversificar las interacciones entre los participantes del proceso educativo con el medio y los contenidos (Cabero, 2007). La Web 2.o llegó también para potenciar el aprendizaje social aprovechando el poder de la comunidad, que consiste en aprender con y de otros usuarios, compartiendo conocimientos; crear comunidades de aprendizaje, caracterizadas por un tema o dominio compartido por los usuarios; y el efecto red que consiste en la transición del trabajo individual a la cooperación entre iguales (Cabero, 2007). 
La interactividad y las interacciones a distancia sustentan la pedagogía de la educación en línea y son identificadas por los investigadores como las claves del éxito (Juwah, 2006). Las estrategias educativas con enfoque constructivista son usadas para generar las interacciones necesarias para producir conocimiento en entornos virtuales de aprendizaje. La perspectiva constructivista enfoca su interés en cómo se construye el conocimiento. La construcción del conocimiento está en función de las experiencias previas, de las estructuras mentales y de las creencias que el sujeto que aprende utiliza para interpretar objetos y eventos. El constructivismo no promueve en el alumno la pre-existencia de una realidad externa, sino que clama porque cada individuo construya su propia realidad, resultante de percepción individual de las experiencias del mundo exterior (Jonassen, 1991). Un ambiente educativo propicio para la construcción del conocimiento sucede cuando el aprendiz interactúa con sus pares con el propósito de alcanzar un objetivo académico en común. Según Martín y López (2012, p. 30): “[...] el aprendizaje debe ser un proceso constructivo del conocimiento que el estudiante elabora a través de actividades aprendiendo a resolver situaciones problemáticas en colaboración con otros compañeros".

El aprendizaje colaborativo es una técnica didáctica de aprendizaje activo determinado por las interacciones complejas entre tres grandes elementos: el conocimiento existenteen los alumnos, el contexto que setrate, y el problema a resolver (Tam, 2000). Una herramienta de colaboración usada comúnmente en ambientes virtuales de aprendizaje son los foros. Para el proceso de interacción en los foros, Xin y Feenberg (2006) proponen un modelo pedagógico de cuatro componentes claves: el involucramiento intelectual, el proceso comunicativo, el diálogo y la motivación, y la dinámica de grupo. El involucramiento intelectual consiste en presentar ejemplos, elaborar argumentos y críticas, definir términos y asignar conceptos a situaciones. El proceso comunicativo reside en los supuestos compartidos, un anclaje común que sirve para los antecedentes de la base de comprensión mutua. El diálogo y la motivación van orientados a la motivación intrínseca de participar con los miembros del grupo. Y la dinámica del grupo fomenta el involucramiento de los participantes, la buena voluntad de contribuir y la construcción de confianza para el logro común de la tarea.

El Aprendizaje Basado en Problemas (ABP) es una técnica didáctica con raíces en las escuelas de medicina de Case Western Reserve University en los Estados Unidos en los años cincuenta y en McMaster University en Canadá en los años sesenta. Estas escuelas cuestionaron qué tan bien los cursos en ciencias clínicas entrenaban a los médicos en las habilidades de solución de problemas y como gestores de su propio aprendizaje (Uden y Beaumont, 2006). Para Barrows (1996) el ABP es un método de 
aprendizaje basado en el principio de usar problemas como punto de partida para la adquisición e integración de los nuevos conocimientos. Sin embargo, a más de medio siglo del desarrollo del ABP, aún existen diferentes posturas e incluso algunas ambigüedades sobre la conceptualización del ABP. Para Vernon y Blake (1993) el ABP es algo más que un método de enseñanza, es una mezcla compleja de filosofía general de enseñanza, objetivos de aprendizaje y metas. De manera similar, Hidalgo et al. (2008) conciben el ABP como una filosofía del proceso educativo, como un paradigma curricular, y como una estrategia educativa y metodológica. Los programas de ABP usan material que atrae el interés de los estudiantes hacia un problema. Los problemas son presentados en el mismo contexto en que pueden ser encontrados en situaciones del mundo real. Al resolver un problema, el aprendizaje también cruza los límites disciplinarios tradicionales; los estudiantes deben identificar las cuestiones que deben aprender para poder resolver el problema y trabajan cooperativamente en grupos pequeños, incluyendo un profesor que actúa como facilitador para ayudarlos en su proceso de aprendizaje (Uden y Beaumont, 2006).

Desarrollado por la Facultad de Medicina en la Universidad de Alcalá, el modelo ABP 4x4, denominado así por su versatilidad y aptitud todo terreno, consta de 4 pasos (AIRE: Análisis inicial, Investigación, Resolución y Evaluación) y se realiza en cuatro escenarios de trabajo: clase completa, grupo sin tutor, tutoría en grupo $\mathrm{y}$ trabajo individual. La fortaleza del modelo $\mathrm{ABP} 4 \mathrm{x} 4$ reside en un entrenamiento metodológico inicial eficaz realizado con la clase completa y unas normas e instrucciones claras. Los grupos trabajan intensivamente sin intervención del profesor y fuera del horario de clases. La mayor parte del trabajo del alumno se realiza en ausencia del profesor (grupo sin tutor y trabajo individual) disminuyendo el tiempo que el tutor debe dedicar a cada grupo. Esta característica permite aplicar el método en grandes números de alumnos, manejando por cada tutor hasta 25 grupos y hasta 100 alumnos (Prieto et al., 2006). El modelo ABP 4x4 simplifica en 4 pasos el modelo de 7 pasos de Maastrich. La tabla 1 presenta un resumen de las actividades por fase y rol que integran del modelo ABP 4x4: 


\begin{tabular}{|c|c|c|c|}
\hline & Fase & Profesor / Tutor & Estudiantes \\
\hline 1. & $\begin{array}{l}\text { Activación del } \\
\text { conocimiento y } \\
\text { análisis. }\end{array}$ & $\begin{array}{ll}\text { - } & \text { Forma grupos. } \\
\text { - } & \text { Presenta el problema. } \\
\text { - } & \text { Activa los grupos. } \\
\text { - } & \text { Supervisa el plan. }\end{array}$ & $\begin{array}{ll}\text { - } & \text { Activación del conocimiento. } \\
\text { - } & \text { Tormenta de ideas. } \\
\text { Identificar elementos del } \\
\text { problema, cuestiones, guía e } \\
\text { hipótesis. }\end{array}$ \\
\hline 2. & Investigación. & $\begin{array}{l}\text { - } \quad \text { Dirige a recursos. } \\
\text { Proporciona } \\
\text { instrucción y } \\
\text { retroalimentación. }\end{array}$ & $\begin{array}{l}\text { - Usan las cuestiones claves } \\
\text { para orientar su búsqueda de } \\
\text { información. } \\
\text { - Organizan la información. } \\
\text { - Definen el problema. }\end{array}$ \\
\hline & $\begin{array}{l}\text { Reanálisis y } \\
\text { Resolución: } \\
\text { consideración } \\
\text { de soluciones e } \\
\text { informe. }\end{array}$ & $\begin{array}{ll}\text { - } & \text { Exige soluciones. } \\
\text { Encarrila a los } \\
\text { descarrilados. }\end{array}$ & $\begin{array}{l}\text { Piensan, discuten y vuelven } \\
\text { a buscar. } \\
\text { Diseñan soluciones para el } \\
\text { problema. } \\
\text { - Las transmiten por escrito. }\end{array}$ \\
\hline & $\begin{array}{l}\text { Evaluación: } \\
\text { reflexión } \\
\text { metacognitiva. }\end{array}$ & $\begin{array}{l}\text { Dirigen discusión y } \\
\text { reflexión grupal. } \\
\text { Evalúa el desempeño } \\
\text { de las competencias. }\end{array}$ & $\begin{array}{l}\text { Presentan sus soluciones } \\
\text { al resto de la clase y las } \\
\text { discuten. } \\
\text { - } \quad \text { Evalúan la actividad. }\end{array}$ \\
\hline
\end{tabular}

Tabla 1. Método AIRE para el ABP $4 \times 4$

Extraido de: "Un nuevo modelo de aprendizaje basado en problemas, el ABP 4x4 es eficaz para desarrollar competencias profesionales valiosas en asignaturas con más de 100 alumnos", por Prieto et al. (2006)".

\section{METODOLOGÍA}

El diseño de investigación seleccionado para este estudio fue el método de estudio de casos con un enfoque cualitativo, particularmente con perspectiva naturalista (Naturalistic Inquiry), además de ser un estudio de tipo exploratorio. El método de estudio de casos dentro del marco de la investigación cualitativa es conceptualizado por Anguera (1985, p. 80) como: “[...] un examen de un fenómeno específico, como un programa, un evento, una persona, un proceso, una institución o un grupo social. Un caso puede seleccionarse por ser intrínsecamente interesante y lo estudiamos para obtener la máxima comprensión del fenómeno". El objeto o propósito de los estudios de casos cualitativos, según Pérez Serrano (2004, p. 81) consiste en "[...] comprender el significado de una experiencia"; Hernández et al. (2006, p. 20) mencionan que "[...] gran parte de los estudios de caso de este tipo tienen como objetivo documentar una experiencia o evento en profundidad o entender un fenómeno desde la perspectiva de quienes lo vivieron". Con relación a la 
temporalidad aplicable en el método de estudio de casos, Pérez Serrano (2004, p. 87) sostiene que "este estudio se puede realizar, considerando al sujeto en un momento concreto y empleando las más diversas técnicas”.

La implementación del estudio de caso de esta investigación se realizó conforme a las fases propuestas por Pérez Serrano (2004):

- La fase preactiva corresponde al trabajo realizado durante el desarrollo de la definición del problema, el marco teórico, la metodología y el diseño de la actividad académica basada en ABP.

- La fase interactiva o trabajo de campo se realiza en un periodo de tres semanas dedicadas a impartir en línea y a distancia la actividad académica basada en la técnica didáctica ABP. Durante esta fase el investigador interviene simultáneamente en dos roles: como facilitador de la actividad y como observador participante recolectando información en el diario de campo, analizando y actualizando los ficheros de evidencia documental. Al finalizar la actividad académica en línea, retoma su rol de investigador, realiza entrevistas a los participantes, recolecta datos en bruto y procede con el análisis de los datos capturados durante esta fase.

- El estudio de caso finaliza en la fase pos activa con la redacción del reporte de resultados, el mismo que es sometido a un proceso de reflexión crítica de los resultados con el propósito de emitir una serie de observaciones y recomendaciones a plasmar en el reporte final de esta investigación.

\section{OBJETO DE ESTUDIO}

La empresa Éxito Software está ubicada en Los Mochis, Sinaloa, México. Emplea a más de 30 personas para desempeñar funciones operativas y administrativas. La formación académica de su personal se muestra en la siguiente tabla:

\begin{tabular}{l|l|l|l}
\hline Escolaridad & Hombres & Mujeres & Total \\
\hline Técnico profesional & 1 & 1 & 2 \\
\hline Licenciatura & 18 & 10 & 18 \\
\hline Maestría & 3 & 0 & 3 \\
\hline Total & 22 & 11 & 23 \\
\hline
\end{tabular}

Tabla 2. Dispersión de personal por escolaridad y género 
Éxito Software cuenta con una red de distribuidores ubicados en las principales ciudades de la república mexicana (Cd. de México, Guadalajara, Monterrey, Veracruz, Puebla, Tijuana, Hermosillo, Cd. Juárez, Chihuahua, Durango, Torreón, León, Aguascalientes, Oaxaca, Tapachula, Mérida, Cancún).

\section{POBLACIÓN Y MUESTRA}

La población participante en este estudio estuvo conformada por un instructor y seis alumnos que son implementadores de soluciones de Éxito Software.

Sus datos demográficos son: 6 participantes de sexo masculino; dispersión de edades: 2 personas menores a 30 años y 3 personas entre 31 y 40 años y una persona mayor a 40 años; formación académica: 1 licenciado en física, 3 licenciados en informática y 2 ingenieros en sistemas computacionales. Los 6 participantes poseen competencias informáticas, definidas por Valerio y Valenzuela (2012, p. 143) como "[...] habilidades requeridas para interactuar en un ambiente electrónico", y consisten en competencias básicas en computación incluyendo tecnología web 2.0 como el manejo de foros, wikis, el correo electrónico y la mensajería síncrona.

Con relación al perfil de los participantes, en este estudio participan implementadores desoluciones de ÉxitoSoftware (personal desoporte, distribuidores y administradores de sistemas). El personal de soporte labora bajo contrato en Éxito Software dentro del área de asistencia técnica; su función principal consiste en capacitar, dar asesoría y resolver dudas técnicas y operativas en ServoEscolar y SesWeb al usuario final en las instituciones educativas; estos servicios son prestados de manera presencial, telefónica o electrónica (e-mail, skype o chat). Los distribuidores laboran en empresas independientes representando a Éxito Software en una región (ciudad o estado). Sus funciones son: promocionar, vender, capacitar y prestar asistencia técnica en los sistemas ServoEscolar y SesWeb. Los administradores de sistemas laboran en el departamento de informática de una institución educativa que contrató la plataforma SesWeb; su función principal consiste en personalizar la plataforma al contexto de la organización educativa, sincronizar los datos entre ServoEscolar y SesWeb, así como capacitar, dar asesoría y resolver dudas técnicas a los usuarios en su institución educativa. Aunque el contexto donde se desempeñan los implementadores es distinto, el común denominador son las funciones de asesor $\mathrm{y}$ capacitador ante el usuario final.

Para realizar la actividad académica basada en la técnica educativa ABP aplicando el modelo ABP 4x4, se seleccionó de manera no aleatoria a seis personas 
como participantes del estudio (un muestreo intencional). El tamaño de la muestra fue pequeño. Los seis participantes integraron dos grupos de tres personas cada uno, lo que constituyó la población en estudio y por tanto la muestra seleccionada. Por la naturaleza de la presente investigación, que fue de corte cualitativo, el interés se centró en un análisis a profundidad y micro de individuos que mostrarían los elementos más significativos del fenómeno o evento educativo analizado, es decir, poder estudiar a grupos pequeños de personas para poder analizarlos con más detenimiento debido a la riqueza social de elementos diversos que presentan (económicos, políticos, ideológicos, culturales, etc.), siendo esta una característica típica de los métodos cualitativos, los cuales no buscan generalizaciones sino respetar y representar en lo posible la naturaleza única del fenómeno o evento social y educativo investigado. Esto podría ser percibido como un sesgo por parte de las posturas axiológicas de paradigmas teóricos que buscan la generalización y la objetividad, sin tomar en cuenta la complejidad, el contexto y la originalidad única y muchas veces subjetiva e irrepetible del fenómeno social (Lincoln y Guba, 1985). Martínez Migueles (2006, p. 86) comenta con respecto a cómo se maneja el muestreo en los métodos cualitativos, lo siguiente:

"Cada uno de los métodos cualitativos [existentes] [...] tiene su forma propia de entender la muestra que nos ofrecerá la información necesaria para realizar la investigación. Pero, en general, la opción ontológica asumida por todos ellos (que es estructural-sistémica) nos exige una muestra que no podrá estar constituida por elementos aleatorios, escogidos al azar y descontextualizados (como es, la mayoría de las veces, la información recogida a través de encuestas o cuestionarios preconcebidos), sino por "un todo" sistémico con vida propia, como es una persona, una institución, una etnia, un grupo social, etc. Por ellos, se impone la muestra intencional, donde se prioriza la profundidad sobre la extensión, y la muestra se reduce en su amplitud numérica. Sin embargo, conviene escogerla de forma que estén representadas de la mejor manera posible las variables de sexo, edad, nivel socioeconómico, profesión, etc., según el caso, y que su información puede ser diferente y hasta contrastante".

\section{El mismo autor comenta también:}

"Como la muestra estudiada incide decisivamente en los resultados que se obtendrán, es importante elegirla muy cuidadosamente. Toda información será, después, interpretada en el marco de referencia o situación que la generó. Los tipos de muestra son, básicamente, dos: la muestra estadística o probabilística y la muestra intencional o basada en criterios (la cualitativa). [...] En la muestra intencional se elige una serie de criterios que se consideran necesarios o altamente convenientes para tener una unidad de análisis con las mayores ventajas para los fines que persigue la investigación. Por ellos suelen eliminar los casos atípicos o muy peculiares y calibrar muy bien la influencia de todo lo que tiene carácter excepcional; sin embargo, se procura que la muestra represente lo mejor posible los subgrupos naturales, [...] y que se complementen y equilibren. Es 
decir, se trata de buscar una muestra que sea comprensiva y que tenga, a su vez, en cuenta los casos negativos o desviantes, pero haciendo énfasis en los casos más representativos y paradigmáticos y explotando a los informantes clave (personas con conocimientos especiales, estatus y buena capacidad de información)" (Martínez Migueles, 2006, p. 86).

En esta investigación la muestra fue intencional y constituyó el estudio de caso, en donde se aplicó y experimentó la técnica didáctica $\mathrm{ABP}$, que se describirá más adelante. Hernández, et al. (2006, p. 564) dejan en claro que "incluso, la muestra puede ser una sola unidad de análisis (por ejemplo, un estudio de caso)”.

\section{DISEÑO DE LA ACTIVIDAD ACADÉMICA ABP QUE FUE OBSERVADA}

La actividad académica a desarrollar y explorar en este trabajo de investigación se denomina "Módulo de programación de cursos en SesWeb". Esta actividad pertenece al curso "Temas selectos de SesWeb" cuyo propósito es capacitar al personal de soporte y distribuidores en la nueva funcionalidad incorporada a la plataforma de SesWeb. Este curso forma parte del programa de capacitación de Éxito Software y se ofrece como un recurso de capacitación en línea en la plataforma de Éxito Software University.

\section{IMPLEMENTACIÓN DE LA ACTIVIDAD ABP}

Para implementar el trabajo académico en línea, se usó la plataforma de servicios educativos SesWeb personalizada para Éxito Software University; las tecnologías de entrega utilizadas fueron: foros, wikis, servicio de aula virtual y encuestas. En las sesiones presenciales participaron localmente personal de soporte de Éxito Software e implementadores ubicados en foráneas. Para virtualizar estas sesiones presenciales se usó la herramienta de webconference Goto Meeting. Para las reuniones síncronas de equipo, los participantes usaron Skype. Como evidencia de estas reuniones el instructor solicitó a los equipos subir a la plataforma el texto de sus conversaciones.

El contenido de esta actividad trata de un contexto educativo simulado (Universidad Tecnológica XYZ), donde la implementación del aula virtual y el módulo de programación de cursos de $S e s W e b$ podría requerirse. La metodología usada para impartir esta actividad en línea se basa en el modelo ABP 4x4, el cual se inicia con una sesión de capacitación grupal en el modelo ABP 4x4, prosigue con el análisis individual de la situación problemática, continúa con la elaboración por equipo del reporte de la primera puesta en común donde los alumnos exponen sus dudas al profesor. El profesor imparte su primera asesoría en línea interactuando 
con los alumnos en los foros y evalúa y retroalimenta el reporte de la primera puesta a punto. Los alumnos proceden con el desarrollo de la presentación de su solución, la misma que es evaluada por el profesor en una segunda asesoría, donde el equipo recibe la retroalimentación indicando oportunidades de mejoras y su visto bueno para su presentación final. Finalmente, en sesión grupal, los equipos exponen sus soluciones recibiendo comentarios del profesor y los alumnos del otro equipo. La evaluación académica se realiza en cuatro momentos: al entregar el reporte de la primera puesta en común, en la presentación del borrador de la solución, en la presentación final de la solución y cuando los alumnos realizan una co-evaluación del desempeño de los integrantes de su equipo. Finalmente, para conocer las actitudes del alumno hacia la actividad académica, se aplica como herramienta auxiliar un cuestionario auto administrado en línea.

\section{CRONOGRAMA DE IMPLEMENTACIÓN}

La implementación de esta actividad académica se realizó en tres semanas conforme a este programa:

\begin{tabular}{|c|c|c|c|}
\hline Semana & Actividad & Modalidad & Fecha \\
\hline 1 & $\begin{array}{ll}\text { - } & \text { Capacitación en el modelo ABP } 4 \times 4 . \\
\text { - } & \text { Capacitación en búsqueda de } \\
\text { información en fuentes digitales. } \\
\text { - } \quad \text { Exposición del problema. }\end{array}$ & Presencial & 18 ene. \\
\hline 1 & $\begin{array}{ll}\text { - } & \text { Análisis individual del problema. } \\
\text { - } & \text { Primera puesta en común. } \\
\end{array}$ & $\begin{array}{l}\text { En línea } \\
\text { En línea }\end{array}$ & $\begin{array}{l}19-20 \text { ene. } \\
21 \text { ene. }\end{array}$ \\
\hline 1 & - $\quad$ Primera sesión de asesoría. & En línea & 24 ene. \\
\hline 2 & $\begin{array}{ll}\text { Elaborar la presentación de la } \\
\text { solución. }\end{array}$ & En línea & 25-27 ene. \\
\hline 2 & $\begin{array}{l}\text { - Segunda puesta en común, para } \\
\text { integrar, revisar y corregir la } \\
\text { presentación. }\end{array}$ & En línea & 28 ene. \\
\hline 2 & - $\quad$ Segunda sesión de asesoría. & En línea & 31 ene. \\
\hline 3 & - $\quad$ Presentación de soluciones. & Presencial & 2 feb. \\
\hline 3 & $\begin{array}{l}\text { - } \quad \text { Coevaluación. } \\
\text { - Encuesta de actitudes. }\end{array}$ & $\begin{array}{l}\text { En línea } \\
\text { En línea }\end{array}$ & $\begin{array}{l}3 \text { feb. } \\
4-5 \text { feb. }\end{array}$ \\
\hline 3 & $\begin{array}{l}\text { - } \quad \text { Presentación de resultados. } \\
\text { Entrega de reconocimientos y } \\
\text { agradecimiento. }\end{array}$ & Presencial & 7 feb. \\
\hline
\end{tabular}

Tabla 3. Resumen de actividades por semana 


\section{FUENTES DE INFORMACIÓN Y TÉCNICAS DE RECOLECCIÓN DE DATOS}

Las fuentes de información consultadas y analizadas para recopilar datos en esta investigación provienen de tres orígenes: 1) consulta directa a los alumnos mediante entrevistas y como herramienta de apoyo adicional se aplicó un cuestionario autoadministrado en línea al concluir el trabajo académico, 2) análisis de evidencias del trabajo académico en línea obtenido de las bitácoras de acceso a la plataforma, la revisión del trabajo académico colaborativo en foros y wikis, las grabaciones de vídeo de las sesiones síncronas, los audios de sesiones en Skype y registros de conversaciones de chat, y 3) la opinión del investigador como observador asentada en las anotaciones del diario de reflexión.

Las técnicas de recolección de datos utilizados en este trabajo de investigación fueron la entrevista cualitativa y la observación participante. Los datos recolectados aplicados en ambas técnicas fueron registrados en los cuadernos de trabajo de campo y diarios de reflexión.

\section{La observación}

La observación es la técnica de recolección de datos comúnmente usada durante el proceso de inmersión inicial de una investigación cualitativa (Hernández et al., 2006). El investigador entiende a los participantes, no únicamente registra hechos (Williams et al., 2005). En esta investigación la técnica de observación cualitativa se aplicó en dos modalidades: observación participante y observación virtual. La primera modalidad se aplicó en las sesiones presenciales, mientras que la observación virtual se realizó para recolectar evidencias del trabajo colaborativo derivado de la actividad académica en línea, tal evidencia se obtuvo del texto escrito en foros y wikis, de bitácoras de conversaciones y grabaciones de audio de reuniones en Skype.

\section{La entrevista}

La entrevista cualitativa se caracteriza por ser más íntima, flexible y abierta. Esta se define como una reunión para intercambiar información entre el entrevistador y el entrevistado (Hernández et al., 2006). Las entrevistas se realizaron al concluir la actividad académica en línea para complementar los datos obtenidos mediante observación participante y observación virtual. El formato de entrevista fue semiestructurado, permitiendo al entrevistador realizar preguntas adicionales a las consideradas en la guía de entrevista. Para capturar con detalle las respuestas la entrevista se grabó en audio para su transcripción y análisis posterior. 


\section{Análisis y recolección de datos}

En investigaciones que utilizan el enfoque cualitativo la recolección (captura) y el análisis de los datos ocurren prácticamente en paralelo. Al recolectar datos, el proceso esencial consiste en dar una estructura a datos no estructurados provenientes de narraciones de participantes como textos escritos y anotaciones en la bitácora de campo (Hernández et al., 2006). En esta investigación los datos recolectados mediante la observación virtual provenían de los espacios de colaboración de la plataforma: foros, wikis, transcripciones de audio y bitácoras de conversaciones de reuniones por Skype. Estos datos fueron complementados con información obtenida de las entrevistas y de un cuestionario autoadministrado. Los datos provenientes de las tres fuentes anteriores fueron organizados en segmentos y analizados hasta su saturación, identificando unidades de análisis. Las unidades de análisis obtenidas fueron a su vez agrupadas en componentes y categorías relacionadas con las variables principales de esta investigación: la técnica didáctica ABP y el aprendizaje colaborativo. Para unificar la presentación de los datos obtenidos por fuente y facilitar el análisis de datos se creó esta estructura:

\begin{tabular}{|c|c|c|}
\hline Categorías & Componente & Unidad de análisis \\
\hline \multirow[t]{7}{*}{$\begin{array}{l}\text { Aprendizaje } \\
\text { colaborativo. }\end{array}$} & \multirow[t]{3}{*}{ Trabajo colaborativo. } & Organización del equipo. \\
\hline & & Aportaciones individuales. \\
\hline & & Integración de entregables. \\
\hline & \multirow[t]{2}{*}{ Interacciones alumno-alumno. } & Comunicación interna del equipo. \\
\hline & & Conciliación de diferentes puntos de vista. \\
\hline & \multirow[t]{2}{*}{ Interacciones profesor-alumno. } & Atención a dudas. \\
\hline & & $\begin{array}{l}\text { Revisión y retroalimentación de } \\
\text { entregables. }\end{array}$ \\
\hline \multirow[t]{7}{*}{$\begin{array}{l}\text { Técnica } \\
\text { didáctica } \\
\text { ABP. }\end{array}$} & \multirow[t]{4}{*}{ Beneficios de aplicar la técnica ABP. } & Ambiente de aprendizaje estimulante. \\
\hline & & Interacciones entre diferentes disciplinas. \\
\hline & & Colaboración entre estudiantes. \\
\hline & & Más contacto docente con estudiantes. \\
\hline & \multirow{3}{*}{$\begin{array}{l}\text { Problemas u obstáculos. } \\
\text { Contribuciones al proceso aprendizaje. }\end{array}$} & Problemas identificados. \\
\hline & & Fomenta aprendizaje profundo. \\
\hline & & Fomenta aprendizaje autodirigido. \\
\hline
\end{tabular}

Tabla 4. Estructura de análisis de datos 
La información recolectada mediante los procesos de observación virtual, el cuestionario autoadministrado en línea y en las entrevistas, se presenta agrupada por categorías en las tablas 5 y 6 .

\begin{tabular}{|c|c|c|}
\hline Observación virtual & Cuestionario & Entrevistas \\
\hline $\begin{array}{l}\text { Los alumnos usan foros } \\
\text { para acordar sus fechas de } \\
\text { reunión. }\end{array}$ & $\begin{array}{l}\text { El 100\% de los alumnos } \\
\text { valoran como excelente el } \\
\text { aprendizaje colaborativo } \\
\text { en su equipo. }\end{array}$ & $\begin{array}{l}\text { Tuvieron menos de una semana } \\
\text { para adaptarse y lograr trabajar } \\
\text { como equipo. }\end{array}$ \\
\hline $\begin{array}{l}\text { Las reuniones síncronas de } \\
\text { equipos usan audio y chat } \\
\text { de Skype. }\end{array}$ & $\begin{array}{l}\text { Consideran que no } \\
\text { hubieran obtenido los } \\
\text { mismos resultados } \\
\text { estudiando por su cuenta. }\end{array}$ & $\begin{array}{l}\text { Acuerdan como sistema de trabajo } \\
\text { utilizar Skype, foros y wikis en } \\
\text { SesWeb. }\end{array}$ \\
\hline $\begin{array}{l}\text { Los alumnos en sesiones } \\
\text { síncronas por Skype } \\
\text { resuelven sus dudas, } \\
\text { discuten aportaciones } \\
\text { y alcanzan la puesta en } \\
\text { común de sus ideas. }\end{array}$ & & $\begin{array}{l}\text { En las juntas de equipo discutían } \\
\text { las actividades por hacer, } \\
\text { distribuían su trabajo y publicaban } \\
\text { en foros sus aportaciones para } \\
\text { debatirlas. }\end{array}$ \\
\hline $\begin{array}{l}\text { Los alumnos publican en } \\
\text { foros los productos de } \\
\text { sus reuniones en Skype } \\
\text { y suben sus audios en } \\
\text { buzones FTP. }\end{array}$ & & $\begin{array}{l}\text { Los alumnos se sienten observados } \\
\text { por sus iguales, esto los motivó } \\
\text { a presentar aportaciones bien } \\
\text { argumentadas. }\end{array}$ \\
\hline $\begin{array}{l}\text { Los alumnos suben y } \\
\text { discuten sus aportaciones } \\
\text { en foros. }\end{array}$ & & $\begin{array}{l}\text { Las aportaciones individuales } \\
\text { generan una mezcla de ideas, hasta } \\
\text { armar ideas consistentes. }\end{array}$ \\
\hline $\begin{array}{l}\text { Los alumnos publican en } \\
\text { wikis los entregables de las } \\
\text { dos primeras fases de la } \\
\text { actividad. }\end{array}$ & & $\begin{array}{l}\text { El análisis de las aportaciones es el } \\
\text { momento clave del aprendizaje de } \\
\text { los alumnos. }\end{array}$ \\
\hline \multirow[t]{3}{*}{$\begin{array}{l}\text { Las interacciones alumno- } \\
\text { profesor son vía foros, para } \\
\text { atender dudas y evaluar. }\end{array}$} & & $\begin{array}{l}\text { Aprenden a conciliar diferencias, a } \\
\text { escuchar las opiniones y entender } \\
\text { los puntos de vista de los demás. }\end{array}$ \\
\hline & & $\begin{array}{l}\text { La participación del profesor } \\
\text { es observante, no invasiva e } \\
\text { intervenía cuando era necesario. }\end{array}$ \\
\hline & & $\begin{array}{l}\text { El profesor está atento a sus } \\
\text { dudas, revisa trabajos indicando } \\
\text { mejoras y orientando cómo } \\
\text { continuar. }\end{array}$ \\
\hline
\end{tabular}

Tabla 5. Información recolectada por instrumento en la categoría Aprendizaje Colaborativo 


\begin{tabular}{|c|c|c|}
\hline Observación virtual & Cuestionario & Entrevistas \\
\hline $\begin{array}{l}\text { Los alumnos resuelven } \\
\text { sus dudas entre ellos. }\end{array}$ & $\begin{array}{l}\text { El 100\% de los alumnos } \\
\text { califican como excelente } \\
\text { la experiencia de haber } \\
\text { participado en la actividad } \\
\text { basada en ABP. }\end{array}$ & $\begin{array}{l}\text { Los alumnos consideran muy } \\
\text { agradable la experiencia de } \\
\text { aprender con ABP. }\end{array}$ \\
\hline $\begin{array}{l}\text { Los alumnos se } \\
\text { compenetran en un } \\
\text { tema que no dominaban } \\
\text { y logran aprender. }\end{array}$ & $\begin{array}{l}\text { El } 100 \% \text { de los alumnos } \\
\text { consideran que la técnica } \\
\text { ABP los motivó en lo } \\
\text { individual y al equipo. }\end{array}$ & $\begin{array}{l}\text { Como beneficios del ABP } \\
\text { consideran: aprender a razonar } \\
\text { una situación problemática, } \\
\text { analizar críticamente, explorar } \\
\text { información, debatirla hasta } \\
\text { alcanzar una puesta en común. }\end{array}$ \\
\hline $\begin{array}{l}\text { Existe un intercambio } \\
\text { de experiencias entre los } \\
\text { integrantes del equipo. }\end{array}$ & $\begin{array}{l}\text { El 100\% de los alumnos } \\
\text { consideran que el ABP } \\
\text { contribuye al proceso de } \\
\text { aprendizaje. }\end{array}$ & $\begin{array}{l}\text { El problema principal fue } \\
\text { negociar fecha y hora para las } \\
\text { reuniones presenciales por } \\
\text { compromisos profesionales y por } \\
\text { estar geográficamente en tres } \\
\text { husos horarios distintos. }\end{array}$ \\
\hline $\begin{array}{l}\text { El docente tiene } \\
\text { visibilidad del avance } \\
\text { del trabajo académico } \\
\text { mediante los foros y } \\
\text { wikis. }\end{array}$ & $\begin{array}{l}\text { El 100\% de los participantes } \\
\text { valoran como útil al ABP en } \\
\text { su campo profesional. }\end{array}$ & $\begin{array}{l}\text { Como otras aplicaciones al } \\
\text { ABP, se consideran las áreas de } \\
\text { soporte técnico, ventas, análisis } \\
\text { de proyectos de TIC nuevos. }\end{array}$ \\
\hline $\begin{array}{l}\text { Existe ausentismo } \\
\text { temporal de alumnos. } \\
\text { En diferentes momentos } \\
\text { un miembro de cada } \\
\text { equipo se desconectó } \\
\text { de la actividad, aunque } \\
\text { posteriormente se } \\
\text { reincorporaron. }\end{array}$ & & \\
\hline
\end{tabular}

Tabla 6. Información recolectada por instrumento en la categoría Técnica Didáctica ABP

La información expuesta en las tablas anteriores aporta evidencias de lo siguiente:

La comunicación interna en los equipos se realizó mediante mensajes de correo electrónico, interacciones asíncronas en los foros de la plataforma SesWeb, y para las reuniones síncronas de equipo usaron Skype.

El sistema de trabajo académico establecido por los equipos consistió en sostener reuniones de equipo síncronas usando Skype, donde analizaban que tenían que aprender o hacer, se distribuían pendientes, trabajaban individualmente y subían sus aportaciones para comentarlas en los foros o en su reunión de equipo. Este 
sistema de trabajo generó dinámicas de grupo en los equipos al desarrollar el trabajo académico.

Para conciliar diferencias en sus puntos de vista, una dinámica observada en uno de los equipos consistió en que los tres integrantes reconocían estar involucrados en el mismo proceso y con una breve explicación de cada uno, llegaban a un punto donde todos estaban de acuerdo y desahogaban el problema.

La actitud del profesor fue mantener una conducta observante y no invasiva durante el desarrollo del trabajo académico. Monitoreó el avance de los equipos en los foros creados para desarrollar las fases de la actividad académica basada en ABP. El profesor atendió las dudas y orientó a los alumnos durante las fases de la actividad, prestando así, asesoría en línea.

Con relación a la técnica didáctica $\mathrm{ABP}$, la evidencia muestra que los alumnos consideran una experiencia agradable aprender con ABP. Los alumnos consideran que la técnica $\mathrm{ABP}$ mantuvo su motivación en lo individual y en equipo. Los alumnos consideran como beneficios de la técnica ABP el aprender a analizar, buscar información en medios electrónicos y a debatir las aportaciones individuales hasta alcanzar la puesta en común. Los principales obstáculos fueron el manejo del tiempo para acordar y asistir a las reuniones de equipo programadas de manera síncrona.

\section{ANÁLISIS E INTERPRETACIÓN DE RESULTADOS}

La información proveniente de las tres fuentes fue recopilada y analizada en diferentes etapas del trabajo de campo: la observación participante se realizó en paralelo con la actividad académica en línea, mientras que las entrevistas y cuestionario auto administrado se aplicaron al finalizar la actividad académica. La información resultante del proceso de triangulación fue agrupada por categoría y confrontada con el marco teórico buscando entender el fenómeno educativo explorado, emergiendo una serie de hallazgos los cuales se exponen por categoría.

\section{Aprendizaje colaborativo}

Con relación a la categoría de Aprendizaje Colaborativo se detectó lo siguiente:

El involucramiento intelectual de los alumnos se observó mediante la autocrítica entre compañeros de equipo, que contribuyó a que las aportaciones de los alumnos, como ellos mismos afirman, no fuera simplemente subir algo. La posibilidad 
de rechazo de las ideas aportadas por parte del equipo motivó en los alumnos el deseo de hacer contribuciones más elaboradas a través de la argumentación de sus ideas, apoyándose en material en línea o en su experiencia profesional. Para Xin y Feenberg (2006) el involucramiento intelectual consiste en la presentación de ejemplos, elaboración de argumentos, críticas, definición de términos y aplicación de conceptos a situaciones.

La construcción social del conocimiento se hizo evidente durante el análisis de las aportaciones individuales, considerado por los mismos alumnos como el momento clave de su aprendizaje. Los alumnos añaden que las aportaciones individuales generan una mezcla de ideas tomando de todo un poco hasta armar algo realmente. En este sentido, Lozano (2010, p. 155) sostiene que "[...] se construye el conocimiento a través del intercambio intelectual en una comunidad de aprendizaje y existen los tiempos adecuados para interpretar y codificar las aportaciones de los demás, lo cual conduce un replanteamiento de ideas que cristalizarán el aprendizaje personal”.

El proceso comunicativo se hizo evidente dentro de los equipos al conciliar diferentes puntos de vista. Los alumnos desarrollaron la capacidad de escuchar y entender las opiniones de los demás, así como aptitudes conciliatorias al tener que aceptar en ocasiones que su aportación no fuera aceptada por los compañeros. El proceso comunicativo para Lozano (2010, p. 154) "[...] reside en los supuestos compartidos, un anclaje común que sirve para los antecedentes de la base de comprensión mutua".

El estilo de tutoría del profesor durante el desarrollo del curso en línea contrastado con la categorización de Lozano (2005), corresponde al estilo facilitador caracterizado por: a) considerar a sus estudiantes como seres pensantes en proceso de formación, b) indaga si los alumnos enfrentan problemas para ofrecer su ayuda, c) asesora a los estudiantes en los huecos cognitivos que detecta y, d) elabora retroalimentaciones detalladas.

\section{Técnica didáctica ABP}

Respecto a la categoría de utilización de la técnica didáctica ABP destaca lo siguiente:

Los datos recolectados aportan evidencias sobre el intercambio de información entre los miembros de los equipos, producto de sus investigaciones individuales y de su experiencia profesional como implementadores de software. Restrepo (2005, p. 
18) menciona "[...] el ABP activa los conocimientos previos y se mejora el interés en el área específica”. Los alumnos en sus entrevistas comentan que el intercambio de experiencias es lo que hace que se enriquezca el trabajo.

La conexión de los alumnos con el contexto de la situación problemática fomentó un ambiente de aprendizaje estimulante entre los alumnos, al despertar en ellos su interés, el sentido de utilidad, así como posibles beneficios profesionales del esfuerzo académico que realizaban. Cheaney e Ingebritsen (2005, p.13) mencionan "[...] un factor que puede influenciar el aprendizaje del estudiante es el sentido de conexión (o la falta de conexión) con el problema central que los alumnos enfrentan". La aplicabilidad del contexto de la situación problemática planteada ejerce una relación directa con el proceso de aprendizaje del individuo. Dick (1991) y Reigeluth (1989) sostienen que el proceso de adquisición y comprensión de nuevos conocimientos está firmemente embebido con el contexto emocional y social donde el aprendizaje toma lugar.

En el modelo ABP 4x4 la revisión de entregables permite al profesor evaluar el avance académico del equipo y sugerir mediante retroalimentación escrita observacionesy mejoras al trabajo realizado, además deorientar al equipo ala siguiente fase. De acuerdo con Prieto et al. (2006), la labor docente en el modelo ABP 4x4 implica: proporcionar instrucción y retroalimentación, exigir soluciones y encarrilar a los descarrilados. El proceso de instrucción, evaluación y retroalimentación constante proporciona al docente visibilidad para conocer el avance de cada equipo y actuar proactivamente como facilitador, asesorando e ideando los andamios necesarios para que los alumnos construyan su propio conocimiento a partir del análisis y desarrollo de solución de una situación problemática.

Con respecto a los beneficios de la técnica didáctica $\mathrm{ABP}$, los alumnos expresaron que son: el aprender a razonar la problemática, a analizar, a investigar, a aportar y a debatir hasta que llega el entendimiento. Los alumnos consideran el modelo ABP 4x4 como una propuesta práctica, sencilla y corta para llegar a la solución de problemas. Bruner (1973, p. 11) menciona que "es fundamental llevar el aprendizaje humano más allá de la mera información, hacia los objetivos de aprender a aprender y a resolver problemas". Los alumnos consideran la técnica ABP como una herramienta muy buena, muy práctica, donde no es necesario dar tantas vueltas a las fórmulas para obtener un resultado.

Con respecto a los problemas u obstáculos experimentados, las evidencias señalan que hubo alumnos con problemas para administrar sus tiempos para cumplir 
con compromisos profesionales y a la vez participar en la actividad académica. Algunos alumnos no mostraron continuidad en su trabajo académico argumentando en sus entrevistas que su ausencia era atribuible a su trabajo, aunque después todos se reincorporaron a las labores de su equipo y lograron acreditar la actividad académica. Uden y Beumont (2006) mencionan que los alumnos no son tan buenos administrando su tiempo como para reservar el tiempo necesario para completar sus trabajos.

Con relación a las contribuciones al proceso de aprendizaje, una característica del $\mathrm{ABP}$ es que fomenta el aprendizaje profundo. Prieto, et al. (2006, p. 173) mencionan que una pretensión del modelo ABP 4 44 es "que los alumnos cambiasen su actitud hacia el aprendizaje y desarrollasen un abordaje más profundo y activo hacia el mismo y capacidades para pensar críticamente”. Un alumno comentó en entrevista que "[...] un análisis profundo de la situación problemática complementado con la investigación necesaria, te lleva a la resolución”. Otro alumno expresa que a través del ABP: "[...] aprendió a buscar en línea, a ser crítico y no dejarse ir por cualquier cosa que encuentre en Internet".

Con referencia al aprendizaje autodirigido que fomenta el ABP durante el trabajo académico en línea, los alumnos tuvieron que actuar con iniciativa propia al adentrarse en un tema nuevo que ninguno dominaba. En lo individual tuvieron que analizar conocimientos previos que les podrían ser útiles y determinar lo que no sabían sobre el tema para investigar en fuentes externas, sintetizar sus ideas y presentarlas como sus contribuciones al equipo, posteriormente debatirlas hasta llegar a puestas en común que los guiaran hacia la resolución del problema. Prieto et al. (2006) comentan que el modelo ABP 4x4 pretende en los alumnos detectar las lagunas en su conocimiento, delimitar sus necesidades de aprendizaje y auto satisfacerlas buscando información, y procesándola por sí mismos para que, de esta forma, desarrollen su competencia para el aprendizaje autodirigido permanente, tan importante para su vida profesional.

En relación con otros usos o aplicaciones del ABP dentro del contexto de una fábrica de software, los alumnos propusieron que el ABP puede ser usado en el área de soporte técnico, para analizar y resolver de manera colaborativa casos complejos de soporte técnico, en el área de ventas, para el análisis y diseño de nuevos proyectos de implementación. Desde la perspectiva del profesor, el ABP en su modelo 4x4 puede aplicarse al análisis de nuevos sistemas de información, donde la situación problemática a automatizar no esté clara o sea ambigua, en casos donde el cliente sabe que tiene un problema, pero no sabe con precisión cuál es el problema a resolver. Las 
evidencias recolectadas en esta investigación demuestran que el modelo ABP 4x4 puede ser impartido en línea y a distancia y además es aplicable en diversos procesos dentro de una fábrica de software. Prieto et al. (2006) mencionan que el modelo ABP $4 \mathrm{x} 4$ ha demostrado su eficacia formativa y su aplicabilidad todo terreno.

\section{CONCLUSIONES}

En este estudio de investigación tuvo por meta explorar y analizar lo que sucede en un curso de capacitación en plataforma educativa SesWeb cuando se incorpora en línea y a distancia una actividad académica basada en la técnica didáctica ABP. Se expuso lo que sucede cuando se implementa este tipo de actividad y a través de un estudio de caso cualitativo se condujo esta exploración y análisis de sucesos durante el desarrollo del trabajo académico en línea.

Se describió el proceso de aprendizaje colaborativo que se lleva a cabo en los equipos de trabajo al aplicar la técnica didáctica $\mathrm{ABP}$ en un curso impartido en línea. Respecto al proceso de aprendizaje colaborativo, cabe destacar como la dinámica del modelo ABP $4 \times 4$ fomenta las aportaciones individuales, consideradas como detonantes del aprendizaje colaborativo. Los alumnos se sienten observados por sus iguales $\mathrm{y}$, ante la posibilidad de que su idea o aportación fuera rechazada por el equipo, se sienten motivados a elaborar aportaciones fundamentadas con argumentos sólidos basados en la literatura explorada o en su experiencia profesional. El análisis de las aportaciones individuales en las reuniones de equipo es el momento clave para el aprendizaje y cristalizador del involucramiento intelectual; las ideas expuestas en las aportaciones individuales son discutidas en equipo hasta integrar una idea consistente. En las reuniones de equipo también se discuten y despejan dudas entre los integrantes del equipo. El proceso comunicativo se experimenta cuando los alumnos desarrollan la capacidad de escuchar y entender opiniones de los demás; las aptitudes conciliatorias se desarrollan al aceptar en ocasiones que su aportación no fuera aceptada por sus compañeros; al interior de los equipos se desarrollan dinámicas internas para conciliar puntos de vista diferentes y llegar a un punto intermedio donde todos estaban de acuerdo. De esta manera, los equipos viven la experiencia de construir socialmente su propio conocimiento a través del aprendizaje colaborativo.

Las evidencias resultantes de este trabajo de investigación señalan que aplicar la técnica ABP en una actividad académica impartida en línea, propicia las interacciones necesarias para el aprendizaje colaborativo. La dinámica del modelo ABP 4x4 promueve el aprendizaje profundo mediante el razonamiento crítico de la situación 
problemática, el aprendizaje autodirigido mediante el análisis y la investigación individual y guía a los alumnos hacia la resolución del problema a resolver planteado como objetivo en la actividad académica.

Destacan como beneficios de la técnica didáctica $\mathrm{ABP}$ en su modelo $4 \times 4$ el fomento en los alumnos del aprendizaje profundo mediante el razonamiento crítico de una situación problemática y el aprendizaje autodirigido a través del análisis y la investigación individual. Las aportaciones individuales, discutidas y consensuadas en las puestas en común fomentan el involucramiento intelectual, guían incrementalmente a los alumnos hacia la resolución del problema y por consiguiente al logro del objetivo de la actividad académica. Las evidencias presentadas demuestran que la actitud de los alumnos hacia la actividad ABP 4x4 fue positiva, el 100\% de los alumnos valoran como excelente su experiencia de aprendizaje en línea con el modelo ABP 4x4. Estas dos evidencias demuestran que, durante la actividad académica en línea, se creó en la virtualidad un ambiente de aprendizaje agradable a los alumnos y este factor también fomentó las condiciones propicias para alcanzar los objetivos académicos establecidos.

Las oportunidades identificadas para la técnica $\mathrm{ABP}$ en el contexto de una fábrica de software, desde la perspectiva de los alumnos son: en el área de soporte técnico para resolver casos de soporte; en el área de ventas para la definición de soluciones en proyectos nuevos, así como en el área de ingeniería para el análisis de sistemas de información en contextos complejos; y desde la perspectiva del profesor, el ABP aplica en implementaciones donde se perciba un escenario de operación complejo. Los obstáculos o problemas identificados fueron el manejo del tiempo y el ausentismo temporal de los alumnos al trabajo académico.

\section{RECOMENDACIONES}

Con base en los resultados y evidencias de esta investigación que revelan la versatilidad y sencillez de implementación del modelo ABP 4X4 dentro del contexto de capacitación corporativa en una fábrica de software para el sector educativo, se sugiere extender su aplicación al proceso de software. Los procesos de desarrollo de software normalmente son ejecutados por equipos de ingeniería, ciertas prácticas que en ocasiones pueden volverse complejas como el análisis y diseño de casos de pruebas, así como en reuniones de revisión entre pares (peer-review), pueden adoptar el modelo ABP 4x4 como estrategia de solución de problemas. 
Para el trabajo académico en equipo se recomienda, cuando les resulte complicado reunirse de manera síncrona, establecer una estrategia de trabajo asíncrona usando los foros. Otra recomendación basada en la literatura de Uden y Beumont (2006) es incluir dentro de la sesión de capacitación inicial pre-ABP un tema sobre administración de proyectos para que los alumnos conozcan cómo administrar sobrecargas de trabajo y redistribuirlas.

Con relación al modelo ABP 4x4 impartido en línea, se recomienda integrar en la sesión inicial o pre-ABP ejercicios sobre el uso de foros de discusión, para que alumnos nuevos en ambientes virtuales de aprendizaje comprendan el potencial y uso de los foros como herramientas de aprendizaje y trabajo colaborativo en línea. Para implementar dinámicas de aprendizaje en foros, se recomienda aplicar el modelo propuesto por Gunawardena et al. (1997) el cual enfatiza la construcción social del conocimiento y consta de cinco niveles: comparación de información, disonancia e inconsistencia, negociación, poner a prueba, y acuerdos y aplicaciones. Además, como recurso de capacitación a profesores en el modelo ABP 4x4, se recomienda la lectura de Prieto et al. (2006) y, de acuerdo a la audiencia del curso, diseñar ejercicios del modelo ABP 4×4 orientados a la solución de situaciones problemáticas cotidianas. De esta manera, se facilita la comprensión del modelo y se despierta el interés del estudiante por aprender usando esta técnica didáctica.

\section{REFERENCIAS BIBLIOGRÁFICAS}

Anguera, M. T. (1985). Posibilidades de la metodología cualitativa versus cuantitativa. Revista investigación educativa, 3 (6), (127-144).

Barrows, H. (1996). Problem-based learning in medicine and beyond: A brief overview. En: Wilkerson, L.; Gijselaers, W. H. (eds.). Bringing Problem-Based Learning to Higher Education: Theory and Practice. San Francisco: JosseyBass Publishers. (3-12).

Bruner, J. (1973). The Relevance of Education. New York,W.W.: Norton \& Company, Inc.

Cabero, J.; Romero, T. (2007). Diseño y producción de TIC para la formación. Barcelona: Editorial UOC.

Cheaney, J.; Ingebritsen (2005). Problembased learning in an online course: a case study.International Review of Research in Open and Distance Learning, 6 (3). Iowa State University. [en línea] Disponible en: http://www.eric.ed.gov/ ERICWebPortal/contentdelivery/ servlet/ERICServlet?accno=EJ846861 (consulta 2010, 20 de octubre).

Cruz Meléndez, A.; Alfaro Rivera, J. A.; Ramírez Montoya, M. S. (2012). Objeto de aprendizaje abierto para la formación docente orientado a desarrollar competencias de pensamiento crítico con énfasis en habilidades cognitivas. RIED. Revista Iberoamericana de Educación a Distancia, 15 (1), (103125).

Dick, W. (1991). An instructional designer's view of constructivism. Educational Technology, 5, (41-44).

Ehuletche, A. M.; De Stefano, A. (2011). Evaluación de las competencias para 
la formación de tutores de e-learning. RIED. Revista Iberoamericana de Educación a Distancia, 14 (1), (75-86).

García-Famoso, M. (2005). Aprendizaje basado en problemas en "introducción a los computadores". Departamento de ingeniería informática y matemáticas. Universitat Rovira i Virgili: Tarragona. [en línea] Disponible en: http://bioinfo. uib.es/ joemiro/aenui/procJenui/ Jen2005/gaapre.pdf (consulta 2010, 7 de octubre).

Gunawardena, Ch.; Lowe, C.; Anderson, T. (1997). Analysis of a global online debate and the development of an interaction analysis model for examing social construction of knowledge in computer conferencing. Journal of Educational and Computing Research,17 (4), (395429).

Hernández, S. R.; Fernández, C. C.; Baptista, L. P. (2006). Metodología de la investigación. Cuarta edición. México: McGraw Hill.

Hernández, S. R.; Fernández, C. C.; Baptista, L. P. (2006). Metodología de la investigación. [CD-ROM]. Capítulo 4: Estudios de caso. Cuarta edición. México: McGraw Hill.

Hidalgo, O. R.; Gallegos, A. P.; Sandoval, C. G.; Sempértegui, G. M. (2008). Aprendizaje basado en problemas: un salto de calidad en educación médica. Equinoccio series académicas, 5. Quito, Ecuador: Editorial EquaOffset. [en línea] Disponible en: http://www.ute. edu.ec/noticias/equinoccio/ART\%20 II.pdf (consulta 2010, 5 de octubre).

Juwah, C. (2006). Interactions in online education: implications for theory \& practice. New York: Routledge.

Jonassen, D. H. (1991). Objetivism vs. constructivism: do we need a philosophical paradigm shift? Educational Research \& Development, 38 (3), (5-14).
Lincoln, Y.; Guba, E. (1985). Naturalistic Inquiry. San Francisco, CA: SAGE.

López Benavides, D.; Álvarez Valdivia, I. (2011). Promover la regulación del comportamiento en tareas de aprendizaje cooperativo en línea a través de la evaluación. RIED. Revista Iberoamericana de Educación a Distancia, volumen 14 (1), (161-183).

Lozano, R. A. (2010). Moderación y facilitación de espacios de colaboración virtuales: la función del profesor tutor. En: Burgos, J.; Lozano A. (coord.). Tecnología Educativa y redes de aprendizaje de colaboración: Retos $y$ realidades de innovación en el ambiente educativo. México, D. F.: Trillas.

Lozano, R. A. (2005). Identificación de los estilos de tutoreo en cursos en línea desde una perspectiva de teoría emergente. Disertación doctoral no publicada. Universidad Virtual del Tecnológico de Monterrey: Monterrey, México.

Martínez Migueles, M. (2006). Ciencia y arte en la metodología cualitativa: Métodos hermenéuticos, métodos fenomenológicos, métodos etnográficos. México, D.F.: Trillas.

Martín Sánchez, M. A.; López Meneses, E. (2012). La sociedad de la información y la formación del profesorado. E-actividades y aprendizaje colaborativo. RIED. Revista Iberoamericana de Educación a Distancia, 15 (1), (15-35).

Pastore, R. (2002). "E-learning in education: An overview". Proceedings of Society for Information Technology and Teacher Education International Conference 2002, Chesapeake, V. A., AAC, (275-276).

Pérez Serrano, G. (2004). Investigación cualitativa. Retos e interrogantes I. Métodos, 4ta. Edición, Madrid: Editorial La Muralla. 
Prieto, M. A.; Barbarroja, E. J.; Reyes, M. E.; Monserrat, S. J.; Díaz, M. D. (2006). Un nuevo modelo de aprendizaje basado en problemas, el ABP 4X4 es eficaz para desarrollar competencias profesionales valiosas en asignaturas con más de 100 alumnos. Aula Abierta, 87, (171194). Universidad de Oviedo. [en línea] Disponible en: http://dialnet.unirioja. es/servlet/articulo? codigo $=2583970$ (consulta 2010, 7 de octubre).

Reigeluth, C. M. (1989). Educational technology at the crossroads: new mindsets and new directions. Educational Technology Research and Development, 37 (1), (1042-1629).

Restrepo, G. B. (2005). Aprendizaje basado en problemas (ABP): una innovación didáctica para la enseñanza universitaria. Educación y Educadores, 8. (9-19) Universidad de la Sabana Facultad de Educación. [en línea] Disponible en: http://personaybioetica. unisabana.edu.co/index.php/eye/ article/view/562/654 (consulta 2010, 20 de octubre).

Tam Constructivism (2000). Instructional design and Technology: Implications for transforming distance learning. Education technology \& society, 3 (2), (50-60).

Uden, L.; Beaumont, C. (2006). Technology and Problem-Based Learning. Hershey, London, Melbourne, Singapore: Information Since Publishing.

Valerio Ureña, G.; Valenzuela González, J. R. (2011). Competencias informáticas para el e-learning 2.0. RIED. Revista Iberoamericana de Educación a Distancia, 14 (1), (137-160).

Vernon, D. T. A.; Blake, R. L. (1993). Does problem-based learning work? A meta-analysis of evaluative research. Academic Medicine, 68 (7), (550-563).

Williams, M.; Unrau, Y. A.; Grinnell, R. M. (2005). The qualitative research approach. En: Grinell, R. M.; Unrau. Y. A. (Eds.), Social work: Research and evaluation. Quantitative and qualitative approaches. 7ma. Edición, (75-87). Nueva York: Oxford University Press.

Xin, C.; Feenberg, A. (2006). Pedagogy in cyberspace: The dynamics of online discourse. Journal of distanteducation, 21 (2), (1-25).

\section{PERFIL ACADÉMICO Y PROFESIONAL DE LOS AUTORES}

Moisés David Armenta Hernández. Ingeniero en Sistemas Computacionales por el Instituto Tecnológico y de Estudios Superiores de Monterrey Campus Ciudad Obregón, en Sonora, México. Maestro en Tecnología Educativa con acentuación en Capacitación Corporativa por la Universidad Virtual del Instituto Tecnológico y de Estudios Superiores de Monterrey, en Ciudad de Monterrey, México. Arquitecto de soluciones de Software para el sector educativo y Director de proyectos en Éxito Software, en Los Mochis, Sinaloa, México.

E-mail: moisesarmenta@exito.com.mx

Verónica Salinas Urbina. Licenciada en Ciencias de la Educación por la Universidad de Monterrey. Maestra en Educación con especialidad en Desarrollo Cognitivo por el Instituto Tecnológico y de Estudios Superiores de Monterrey. Coordinadora del proceso de evaluación de los cursos ofrecidos por satélite en el 
Tecnológico de Monterrey. Coordinadora de capacitación para profesores y personal de la Universidad Virtual. Asesora en diseño instruccional. Profesora tutora del programa de la Maestría en Tecnología Educativa.

E-mail: veronica.salinas@tecvirtual.mx

Fernando Jorge Mortera Gutiérrez. Licenciado en Antropología Social por la Escuela Nacional de Antropología e Historia. Maestro en Ciencias Sociales con especialidad en Socio-Demografía por la Facultad Latinoamericana de Ciencias Sociales. Doctor en Educación en Educational Human Resource Development con especialidad en educación de adultos y educación a distancia por la Texas A\&M University. Profesor-Investigador de tiempo completo y Director de la Maestría en Tecnología Educativa,de la Universidad Virtual del Tecnológico de Monterrey.

E-mail: fmortera@tecvirtual.mx

DIRECCIÓN DE LOS AUTORES

\begin{abstract}
Moisés David Armenta Hernández, Rafael Buelna 169-4 Pte. Colonia Centro, Los Mochis, Sinaloa, México. C.P. 81200.

Verónica Salinas Urbina, Av. Eugenio Garza Sada 2501 Sur. Colonia Tecnológico. Monterrey, Nuevo León, México. C.P. 64849
\end{abstract}

Fernando Jorge Mortera Gutiérrez, Palancar \#111. Colonia Los Arrecifes, Apodaca,Nuevo León, México. C.P. 66633

Fecha de recepción del artículo: 08/06/2012

Fecha de aceptación del artículo: 17/09/2012

\title{
Como citar este artículo:
}

Armenta Hernández, M. D.; Salinas Urbina, V.; Mortera Gutiérrez, F. (2013). Aplicación de la técnica educativa aprendizaje basado en problemas para capacitación a distancia (e-learning). RIED. Revista Iberoamericana de Educación a Distancia, volumen $16, \mathrm{n}^{0} 1$, pp. 57-83. 